\title{
Crossing Angles of Geometric Graphs
}

\author{
Karin Arikushi1 Csaba D. Tóth $\square^{2}$ \\ 1 Department of Mathematics and Statistics \\ University of Calgary, Calgary, AB, Canada \\ 2 Department of Mathematics \\ California State University Northridge, Los Angeles, CA, USA.
}

\begin{abstract}
We study the crossing angles of geometric graphs in the plane. We introduce the crossing angle number of a graph $G$, denoted can $(G)$, which is the minimum number of angles between crossing edges in a straight-line drawing of $G$. We show that an $n$-vertex graph $G$ with $\operatorname{can}(G)=O(1)$ has $O(n)$ edges, but there are graphs $G$ with bounded degree and arbitrarily large $\operatorname{can}(G)$. We also initiate the study of global crossing angle rigidity for geometric graphs. We construct bounded degree graphs $G=(V, E)$ such that for any two straight-line drawings of $G$ with the same crossing angle pattern, there is a subset $V^{\prime} \subset V$ of $\left|V^{\prime}\right| \geq|V| / 2$ vertices that are embedded into similar point sets in the two drawings.
\end{abstract}

\begin{tabular}{|ccllc|}
\hline Submitted: & Reviewed: & Revised: & Accepted: & Final: \\
Februray 2014 & April 2014 & May 2014 & June 2014 & July 2014 \\
& & Published: & \\
& July 2014 & \\
& Article type: & Communicated by: & \\
& Regular paper & G. Liotta & \\
&
\end{tabular}

A preliminary version of this paper has been presented at the 6th Annual Conference on Combinatorial Optimization and Applications (Banff, AB, 2012) [3. Research supported in part by the NSERC grant RGPIN 35586.

E-mail addresses: karikush@gmail.com (Karin Arikushi) cdtoth@acm.org (Csaba D. Tóth) 


\section{Introduction}

A straight-line drawing of a graph $G=(V, E)$ is a representation of $G$ in which the vertices are mapped into distinct points in the plane, and the edges are drawn as straight-line segments between the corresponding vertices that do not pass through any other vertex. A geometric graph is a graph $G=(V, E)$ together with a straight-line drawing.

The rectilinear crossing number of a graph $G$ (i.e., the minimum number of crossings pairs of edges in any straight-line drawing of $G$ ) has been studied intensely for decades [1. However, angle conditions for the crossing edges have only been recently considered. The motivation comes from cognitive experiments showing that having small crossing angles is negatively correlated to path-tracking ability in a graph drawing [17.

In this paper, we consider two combinatorial aspects of crossing angles in the straight-line drawings of graphs: (1) What is the maximum size of a graph that admits a straight-line drawing with a certain number of different angles between crossing edges? (2) Do the angles between the crossing edges determine the straight-line drawing of a graph (completely or at least partially) up to similarity?
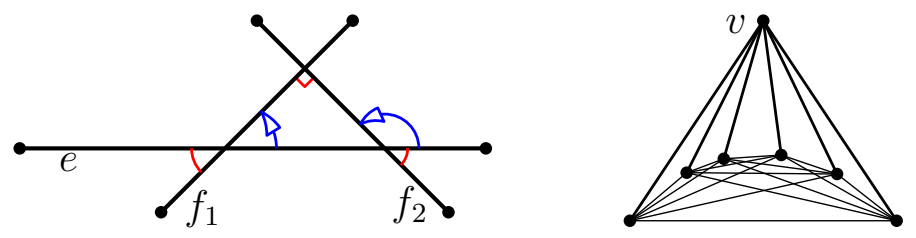

Figure 1: Left: The undirected angles $\angle\left\{e, f_{1}\right\}$ and $\angle\left\{e, f_{2}\right\}$ are both $\pi / 4$, with $\angle\left\{f_{1}, f_{2}\right\}=\pi / 2$. However, the directed crossing angles between these edges are different: we have $\angle\left(e, f_{1}\right)=\pi / 4$ and $\angle\left(e, f_{2}\right)=3 \pi / 4$. Right: A straightline drawing of $K_{7}$. The edges incident to $v$ are crossing free, hence $v$ can be relocated without changing the crossing angles in the drawing.

We define both directed and undirected crossing angles. The (undirected) crossing angle between two crossing edges $e$ and $f$ in a straight-line drawing of a graph is the minimum angle $\angle\{e, f\} \in\left(0, \frac{\pi}{2}\right]$ between the supporting lines of the two edges. The directed crossing angle of two crossing edges $e$ and $f$ is the angle $\angle(e, f) \in(0, \pi)$ such that a counterclockwise rotation through this angle carries the supporting line of $e$ to that of $f$. Refer to Fig. 1)(left). Given a straight-line drawing of an edge $e$ and the (undirected) crossing angle $\angle\{e, f\} \neq \frac{\pi}{2}$, then the edge $f$ may have two possible directions. The drawing of $e$ and the directed crossing angle $\angle(e, f)$ uniquely determine the direction of $f$.

Crossing Angle Number. The crossing angle number of a graph $G$, denoted $\operatorname{can}(G)$, is the minimum number of crossing angles in any straight-line drawing of $G$. In Section 2 , we show that every $n$-vertex graph $G$ has less than $(6 \operatorname{can}(G)+$ $3) n$ edges. We also show that for every $\varepsilon>0$, there are $n$-vertex graphs of maximum degree $O(1 / \varepsilon)$ such that $\operatorname{can}(G)=\Omega\left(n^{1 / 2-\varepsilon}\right)$. 
Global crossing angle rigidity. Motivated by analogous results in rigidity theory, we ask whether the (directed) crossing angles determine a straight-line drawing of a graph uniquely up to similarity. Recall that a straight-line drawing $D=D(G)$ of a graph $G=(V, E)$ is rigid if every straight-line drawing of $G$ with the same (Euclidean) edge lengths as in $D$ is congruent to $D$ (i.e., the drawing $D$ is unique up to congruence). A graph $G=(V, E)$ is globally rigid in the plane if for every function $\ell: E \rightarrow \mathbb{R}^{+}$, any two straight-line drawings of $G$ in which the Euclidean length of each edge $e \in E$ is $\ell(e)$ are congruent. In other words, the edge lengths determine at most one straight-line drawing up to congruence. For instance, it is not difficult to see that every complete graph with 3 or more vertices is globally rigid.

Consider a straight-line drawing $D$ of a graph $G=(V, E)$, and assume we have complete information about the directed crossing angles between the edges: we know which pairs of edges cross, and we also know the directed crossing angle of every two crossing edges. Is this information enough to reconstruct $D$ up to similarity? (Since similarities preserve both angles and the intersection pattern of the edges, the best we can hope for is uniqueness up to similarity.) The answer to this question is negative. If no two edges cross (hence $D$ is a plane drawing), then $D$ is clearly not unique up to similarity. It is not difficult to see that even the complete graphs $K_{n}$ have straight-line drawings that are not uniquely determined by the crossing angles: It is possible that no edge incident to a vertex $v$ crosses any other edges in $D$ (Fig. 1 right), and then $v$ can be relocated without changing the crossing angles. This motivates the definition of global crossing angle rigidity (see below), where we require that at least a constant fraction of the vertices be uniquely determined up to similarity.

Let $G=(V, E)$ be a graph. A directed crossing angle pattern is a function $\alpha: E^{2} \rightarrow[0, \pi) \cup\{\star\}$. We say that a straight-line drawing $D(G)$ is compatible with $\alpha$ if for every two crossing edges, $e$ and $f$, the directed crossing angle is $\angle(e, f)=\alpha(e, f)$; and for every two noncrossing edges, we have $\alpha(e, f)=\star$. We can now define the analogues of rigidity and global rigidity for crossing angles: A straight-line drawing $D_{1}$ of a graph $G$ is crossing angle rigid if in every straight-line drawing $D_{2}$ of $G$, if $D_{1}$ and $D_{2}$ have the same directed crossing angle pattern, then at least $|V| / 2$ vertices are mapped to similar point sets in the two drawings. A graph $G=(V, E)$ is globally crossing angle rigid if for every function $\alpha: E^{2} \rightarrow[0, \pi) \cup\{\star\}$, and for every two straight-line drawings, $D_{1}$ and $D_{2}$, compatible with $\alpha$, there is a vertex set $V^{\prime}(\alpha) \subset V$ of size $\left|V^{\prime}(\alpha)\right| \geq|V| / 2$ that are mapped to similar point sets in the two drawings.

In Section 3, we prove that the complete graph $K_{n}$ is globally crossing angle rigid for $n \geq 24$ (Theorem 2), and we also construct an infinite family of globally crossing angle rigid graphs with maximum degree 47, diameter $O(\log n)$, and $n \geq 24$ vertices (Theorem 3).

Related Work. Previous research on crossing angles focused on the crossing resolution [8, 16] of straight-line (or polyline) drawings, that is, the minimum angle at which crossing edges meet. Didimo et al. 10 consider graphs that 
admit straight-line drawings where crossing edges meet at a right angle. Such drawings are called right angle crossing (for short $R A C$ ) drawings. They prove that every $n$-vertex graph that admits a RAC drawing has at most $4 n-10$ edges, and this bound is best possible. Argyriou et al. [2] show that it is NP-hard to decide whether a given graph admits a RAC drawing. Refer to [7, 9, 14, for recent results on RAC drawings. Note that if a graph $G$ admits a RAC drawing, then its crossing angle number is $\operatorname{can}(G) \leq 1$ (all edge crossings are at angle $\pi / 2$ ). By contrast, we show (Observation 1) that a graph $G$ with $\operatorname{can}(G)=1$ may have $4.5 n-O(\sqrt{n})$ edges. Dujmović et al. [13] generalize RAC-drawings and consider so-called $\alpha$ angle crossing $(\alpha \mathrm{AC})$ graphs, for $0<\alpha \leq \frac{\pi}{2}$. These are straight-line drawings where crossing edges meet at an angle at least $\alpha$. They prove that an $n$-vertex $\alpha \mathrm{AC}$ graph has at most $(\pi / \alpha)(3 n-6)$ edges for $0<\alpha<\pi / 2$ and at most $6 n-12$ edges for $2 \pi / 5<\alpha<\pi / 2$.

The rectilinear crossing number has been studied for decades, but its exact value is not even known for the complete graph [1. It is known, however, that there are families of bounded degree graphs (even cubic graphs [25]) for which the rectilinear crossing number is unbounded [6]. Our results imply (Corollary 2) that there exist families of bounded degree graphs for which the crossing angle number is unbounded.

The crossing angle number is also related to the slope number of a graph $G$, introduced by Wade and Chu [29]. It is the smallest integer $s(G)$ such that $G$ has a straight-line drawing in which the edges have $s(G)$ distinct slopes. The slope number gives an easy upper bound for the crossing angle number, $\operatorname{can}(G) \leq\left(\begin{array}{c}s(G) \\ 2\end{array}\right)$, since the slopes of two crossing edges in a straight-line drawing determine the crossing angle of the two edges. Mukkamala and Pálvölgyi 23] show that every cubic graph has slope number at most 4 . On the other hand, Pach et al. 24] show that there are graphs of maximum degree $d \geq 5$ with arbitrarily large slope numbers. Dujmović et al. [12] improve the lower bound on slope number for $d \geq 9$ and showed that for every $\varepsilon>0$, there are $\Delta$-regular graphs with slope number at least $n^{1-(8+\varepsilon) /(\Delta+4)}$. For planar graphs, Keszegh et al. 22] show that for every $d \in \mathbb{N}$, there is a constant $f(d)=2^{O(d)}$ such that every planar graph with maximum degree at most $d$ admits a straight-line drawing with at most $f(d)$ slopes.

For background information on rigidity theory, refer to the excellent survey by Whiteley [30. Saxe [27] showed that it is strongly NP-hard to decide whether a graph is globally rigid. Jackson and Jordan [18, 20, 21] gave a simple combinatorial characterization of generic global rigidity, where the edge lengths determine at most one straight-line drawing (up to congruence) if the vertices are in general position. They also extended this notion to a so-called length-direction rigidity [19, where each edge has either a prescribed length or a prescribed direction vector. Angle constraints between intersecting circles are considered by Saliola and Whiteley [26], in the context of computer aided design (CAD), and are modeled by distance constraints between points in Euclidean 3-space. 


\section{Graphs with bounded crossing angle numbers}

The geometric thickness [11] of a graph, denoted $\operatorname{gth}(G)$, is the smallest number of layers such that one can draw $G$ in the plane with straight-line edges and assign each edge to a layer so that no two edges on the same layer cross. We establish a relation between the geometric thickness and the crossing angle number.

Theorem 1 For every graph $G$, we have $\operatorname{gth}(G) \leq 2 \operatorname{can}(G)+1$.

Proof: Consider a straight-line drawing $D$ of a graph $G=(V, E)$ with $\operatorname{can}(G)$ crossing angles. We begin by partitioning the edges of $G$ as follows. We define a binary relation on the edges of $G$ where two edges are related if and only if they cross in the drawing $D$. The transitive closure of this relation is an equivalence relation. The set of edges in an equivalence class is a called block of $G$ with respect to $D$. (See Fig. 2 for examples.) We partition the edges of $G$ into blocks.

Let $k=\operatorname{can}(G)$. We partition each block of $G$ into the union of at most $2 k+1$ subsets, each of which is crossing-free in $D$. Let $A=\left\{\alpha_{1}, \ldots, \alpha_{k}\right\}$ denote the set of crossing angles in the drawing $D$. We construct a (possibly infinite) graph $H$ whose vertices are the elements in $A^{\prime}=\left\langle\alpha_{1}, \ldots, \alpha_{k}\right\rangle$, the Abelian group generated by the angles $\alpha_{i}$ where addition is performed modulo $\pi$. Two vertices of $H$ are adjacent if and only if their difference is $\pm \alpha_{i}$ for some $\alpha_{i} \in A$.

For a fixed $\alpha \in A$ and $\beta \in A^{\prime}$, there exists a unique $\beta^{\prime} \in A^{\prime}$ such that $\beta-\beta^{\prime}=\alpha$. Hence, the degree of each $\beta \in H$ is at most $2 k$ and there exists a proper coloring of the vertices of $H$ with at most $2 k+1$ colors. Moreover, each color class is an independent set in $H$. We use the color classes to partition each block of $G$ into planar subgraphs.

Consider a block of $G$ and assume without loss of generality that one edge has slope zero in $D$. Thus, every edge in the block has a direction in $\left\langle\alpha_{1}, \ldots, \alpha_{k}\right\rangle$. (An edge has direction $\alpha \in[0, \pi)$ if it can be rotated clockwise through angle $\alpha$ to horizontal position.) Thus, if $\beta_{i}$ and $\beta_{j}$ are in a color class of $V(H)$, then edges with directions $\beta_{i}$ and $\beta_{j}$ do not cross. We may partition the edges in each block independently to obtain a partition of all edges of $G$ into subgraphs, each of which is planar in the drawing $D$.

We show that every $n$-vertex graph with bounded crossing angle number has $O(n)$ edges. Recall that a planar graph with $n \geq 3$ vertices has at most $3 n-6$ edges.

Corollary 1 A graph $G$ with $n \geq 3$ vertices has at most $(2 \operatorname{can}(G)+1)(3 n-6)$ edges.

Barát et al. [5] proved that for every $\Delta \geq 9, \varepsilon>0$ and $n \in \mathbb{N}$, there is a $\Delta$ regular graph with at least $n$ vertices and geometric thickness $\Omega\left(\sqrt{\Delta} n^{1 / 2-\Delta / 4-\varepsilon}\right)$.

Corollary 2 For every $\Delta \geq 9, \varepsilon>0$ and $n \in \mathbb{N}$, there is a $\Delta$-regular graph $G$ with at least $n$ vertices and $\operatorname{can}(G)=\Omega\left(\sqrt{\Delta} n^{1 / 2-\Delta / 4-\varepsilon}\right)$. 

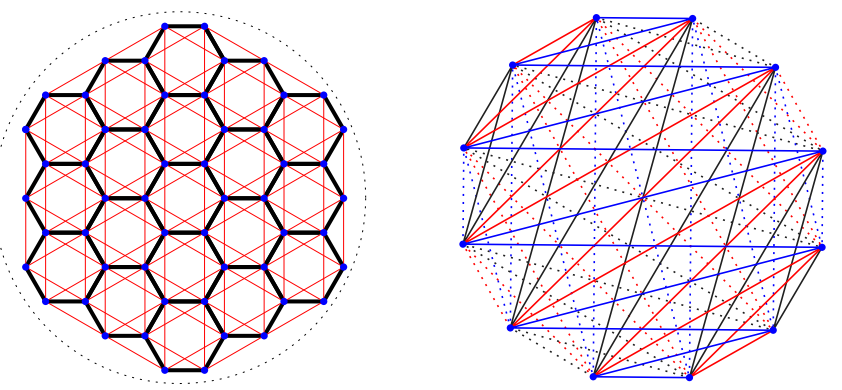

Figure 2: Left: A section of a hexagonal lattice with 54 vertices. The 6 diagonals in each hexagon form a block (even though not all pairs of diagonals cross). Right: A straight-line drawing of $K_{12}$ where the vertices form a regular polygon.

We note here that the class of graphs $G$ with $\operatorname{can}(G)=1$ is strictly larger than the class of graphs that admit RAC drawings (i.e., straight-line drawings in which crossing edges meet at a right angle).

Observation 1 For every $n \in \mathbb{N}$, there exist an $n$-vertex graph $G_{n}$ with $4.5 n$ $O(\sqrt{n})$ edges and $\operatorname{can}(G)=1$.

Proof: We construct an infinite graph $G=(V, E)$. Refer to Fig. 2(left). Let $V$ be the set of points in a hexagonal lattice, and let $E$ consist of the edges of all hexagons, and all 6 diagonals between second neighbors in each hexagon. The degree of every vertex is $3+3 \cdot 2=9$ in $G$. The edges of the hexagons form a plane graph; the diagonals of distinct hexagons do not cross; and two diagonals of the same (regular) hexagon are either parallel or meet at an angle of $\pi / 3$.

Let $o$ be a point in general position with respect to $V$. For every $n \in \mathbb{N}$, let $O_{n}$ be a the smallest disk centered at $o$ that contains exactly $n$ point in $V$; and let $G_{n}$ be the subgraph of $G$ induced by the $n$ vertices in $O_{n}$. The vertex degree is 9 for all but $O(\sqrt{n})$ vertices around the boundary of $O_{n}$. Hence $G_{n}$ has $n$ vertices and $4.5 n-O(\sqrt{n})$ edges.

We also note that the crossing angle number of the $n$-vertex complete graph $K_{n}$ is less than $\frac{n}{2}$.

Observation 2 For $n \geq 2$, we have $\operatorname{can}\left(K_{n}\right) \leq\lfloor n / 2\rfloor-1$.

Proof: We say that an edge of $G$ has direction $\alpha \in[0, \pi)$ if it can be rotated clockwise through angle $\alpha$ to horizontal position. Consider the straight-line drawing of $K_{n}$ such that the vertices are represented by the vertices of a regular $n$-gon with a horizontal side (Fig. 2 right). The set of directions of the edges in this drawing is $\{i \pi / n: 0 \leq i<n\}$. Consequently, the (undirected) angle between the supporting lines of any two edges is in the set $U=\{i \pi / n: 0 \leq$ $i \leq n / 2\}$, where $|U|=\lfloor n / 2\rfloor+1$. However, if the angle between two edges is 0 , then the two edges are parallel; and if the angle is $\pi / n$, then they do not cross. Hence, this drawing of $K_{n}$ has only $|U|-2$ crossing angles, as claimed. 
The bound in Observation 2 is tight for $n=2,3$, and 5 , but $\operatorname{can}\left(K_{4}\right)=0$ and $\operatorname{can}\left(K_{6}\right)=1$. Determining $\operatorname{can}\left(K_{n}\right)$ for $n \geq 7$ is left for future research. For comparison, the slope number of $K_{n}, n \geq 3$, is known to be exactly $n$ [29].

\section{Globally angle-rigid graphs}

Assume that we are given a graph $G=(V, E)$ together with a directed crossing angle pattern $\alpha: E^{2} \rightarrow[0, \pi) \cup\{\star\}$, and we consider straight-line drawings $D(G)$ compatible with $\alpha$. That is, whenever $\alpha(e, f)=\star$, then the relative interiors of the edges $e$ and $f$ are disjoint (although, they may share a common endpoint); and whenever $\alpha(e, f) \in(0, \pi / 2]$, then $e$ and $f$ cross at angle $\alpha(e, f)$. We assume that the position of some vertices is given and we wish to determine the position of additional vertices based on $\alpha$. We start with a simple observation.

Proposition 1 Let $G=(V, E)$ be a graph with edges $p p_{1}, p p_{2}, p_{1} p_{2}, e_{1}, e_{2} \in E$, and let $\alpha$ be a crossing angle pattern such that $\alpha\left(p p_{1}, e_{1}\right) \neq \star$ and $\alpha\left(p p_{2}, e_{2}\right) \neq \star$ (Fig. 3). Then in any straight-line drawing compatible with $\alpha$, the position of $p$ is determined by the slopes of $e_{1}$ and $e_{2}$, and the position of $p_{1}$ and $p_{2}$.

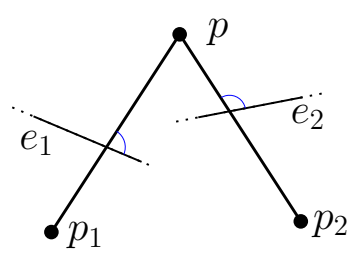

Figure 3: Edges $p p_{1}$ and $p p_{2}$ each cross some other edges of the graph.

Proof: The slope of $e_{1}$ and the crossing angle $\angle\left(p p_{1}, e_{1}\right)$ determine the slope of edge $p p_{1}$. This slope together with the location of $p_{1}$ determines the supporting line of $p p_{1}$. Similarly, $\angle\left(p p_{2}, e_{2}\right)$, the slope of $e_{2}$, and the location of $p_{2}$ determine the supporting line of $p p_{2}$. Note that $p p_{1}$ and $p p_{2}$ cannot be collinear, otherwise an edge would pass through a vertex in the straight-line drawing of $G$. Hence, $p$ is the unique intersection point of the supporting lines of $p p_{1}$ and $p p_{2}$.

\subsection{Tools from Combinatorial Geometry}

Assuming that we already know the position of some of the vertices in a straightline drawing of the complete graph (resp., a complete bipartite graph), in this subsection, we develop basic tools for determining how many points can evade Proposition 1.

Crossing Free and Almost Crossing Free Vertices. We say that a vertex $p$ in a straight-line drawing of a graph is crossing free if no edge incident to $p$ 
(a)

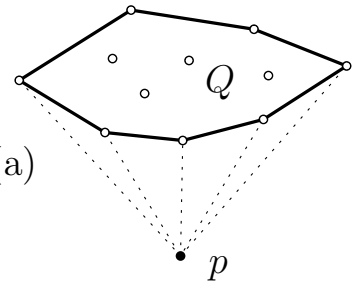

(c)

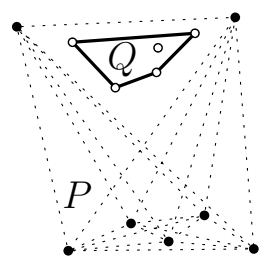

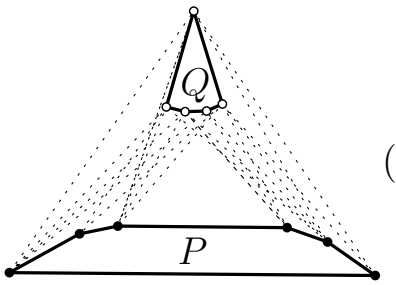

(b)

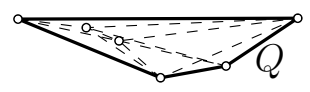

(d)

Figure 4: (a) Point $p$ sees 5 vertices of the convex hull $\operatorname{conv}(Q)$. (b) $P$ and $Q$ are convexly avoiding (but not mutually avoiding). (c) $P$ avoids $Q$ (but $Q$ does not avoid $P$ ). (d) $P$ and $Q$ are mutually avoiding (but not convexly avoiding).

crosses any other edge; and $p$ is almost crossing free if exactly one edge incident to $p$ crosses other edges. A point $p$ sees a vertex $q_{i}$ of a convex polygon $\left(q_{1}, \ldots q_{k}\right)$ if the line segment $p q_{i}$ is disjoint from the interior of $\left(q_{1}, \ldots q_{k}\right)$. Two point sets, $P$ and $Q$, are convexly avoiding if for every $p \in P$ and $q \in Q$, the segment $p q$ is disjoint from the interior of both convex hulls $\operatorname{conv}(P)$ and $\operatorname{conv}(Q)$. See Fig. 4(a)-(b). Note that if $P$ and $Q$ are convexly avoiding, then $P$ and $Q$ are each in convex position. We show that in any drawing of a complete graph with 5 or more vertices, at most one vertex is crossing free or almost crossing free.

Proposition 2 Consider a straight-line drawing of the complete graph $K_{n}=$ $(V, E)$ with $n \geq 5$ vertices. If $p \in V$ is a crossing free vertex in this drawing, then $p$ is a vertex of the convex hull $\operatorname{conv}(V)$, the set $V \backslash\{p\}$ is in convex position, and $p$ sees all vertices of $\operatorname{conv}(V \backslash\{p\})$.

Proof: Suppose that $p$ lies in the interior of $\operatorname{conv}(V)$. By Carathéodory's theorem, there are vertices $q_{1}, q_{2}, q_{3} \in V$ on the boundary of $\operatorname{conv}(V)$ such that $p$ lies in the interior of the triangle $\operatorname{conv}\left(\left\{q_{1}, q_{2}, q_{3}\right\}\right)$. Since $|V| \geq 5$, there is another vertex $r \in V$. The segments $p q_{1}, p q_{2}$ and $p q_{3}$ decompose conv $(V)$ into 3 sectors, and we may assume without loss of generality that $r$ lies in the sector bounded by $p q_{1}$ and $p q_{2}$. The path $p q_{1} \cup p q_{2}$ decomposes $\operatorname{conv}(V)$ into two regions, and points $r$ and $q_{3}$ are in different regions by construction. Hence the segment $r q_{3}$ crosses either $p q_{1}$ or $p q_{2}$. This contradicts our assumption that $p$ is crossing free. Therefore, $p$ is a vertex of the convex hull $\operatorname{conv}(V)$.

Now suppose that $p$ does not convexly avoid $V \backslash\{p\}$. Then there is a vertex $r_{1}$ on the boundary of $\operatorname{conv}(V \backslash\{p\})$ such that $p r_{1}$ intersect the interior of $\operatorname{conv}(V \backslash\{p\})$, and so $p r_{1}$ crosses some edge $r_{2} r_{3}$ on the boundary of $\operatorname{conv}(V \backslash$ $\{p\})$. This contradicts our assumption that $p$ is crossing free. 
Proposition 3 Consider a straight-line drawing of the complete graph $K_{n}=$ $(V, E)$ with $n \geq 6$ vertices. If $p \in V$ is an almost crossing free vertex where edge pq crosses some other edges of the graph, then $p$ is a vertex of $\operatorname{conv}(V)$, $V \backslash\{p, q\}$ is in convex position, and $p$ sees all vertices of $\operatorname{conv}(V \backslash\{p, q\})$.

Proof: By definition, there is exactly one edge incident to $p$, namely $p q$, that crosses other edges. If we delete vertex $q$, we obtain a straight-line embedding of $K_{n-1}, n-1 \geq 5$, where $p$ is crossing free. Proposition 2 completes the proof.

Mutually Avoiding Sets. Let $P$ and $Q$ be two point sets in the plane. We say that $P$ avoids $Q$ if the supporting line of any two points in $P$ is disjoint from $\operatorname{conv}(Q)$; and $P$ and $Q$ are mutually avoiding if $P$ avoids $Q$ and $Q$ avoids $P$. See Fig. 4(c)-(d). Aronov et al. 4] proved that any two point sets, $P$ and $Q$, of size $|P|=|Q|=n / 2$ contain two mutually avoiding subsets $P^{\prime} \subseteq P$ and $Q^{\prime} \subseteq Q$ of size $\left|P^{\prime}\right|,\left|Q^{\prime}\right| \geq \sqrt{n / 24}$. We strengthen their results when $P$ or $Q$ is in convex position.

Proposition 4 Let $P$ and $Q$ be disjoint point sets in the plane such that $|P| \geq 5$ and every $q \in Q$ sees at least $|P|-1$ vertices of $\operatorname{conv}(P)$. Then,

(i) there is a subset $P^{\prime} \subseteq P$ of size $\left|P^{\prime}\right| \geq|P|-3$ in convex position such that $P^{\prime}$ avoids $Q$, and every point $q \in Q$ sees all vertices of $\operatorname{conv}\left(P^{\prime}\right)$; and

(ii) there is a subset $P^{\prime \prime} \subseteq P$ of size $\left|P^{\prime \prime}\right| \geq|P|-1$ in convex position and a subset $Q^{\prime \prime} \subseteq Q$ of size $\left|Q^{\prime \prime}\right| \geq\lceil|Q| / 3\rceil$ such that $P^{\prime \prime}$ avoids $Q^{\prime \prime}$ and every point $q \in Q^{\prime \prime}$ sees all vertices of $\operatorname{conv}\left(P^{\prime \prime}\right)$.
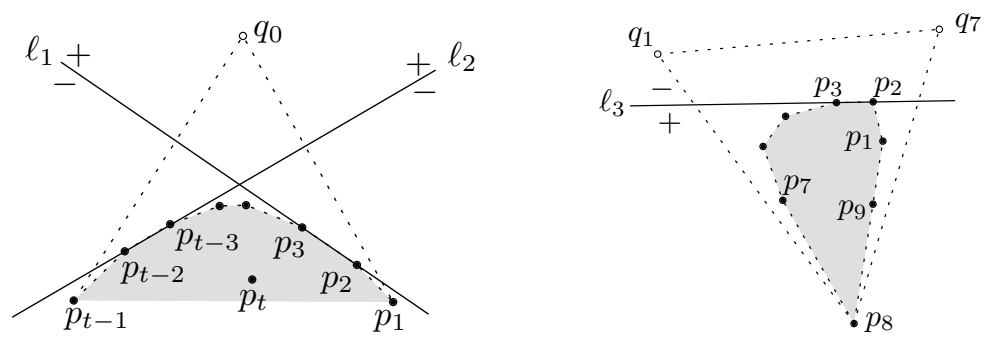

Figure 5: Case 1 (left) and Case 2 (right) in the proof of Proposition 4.

Proof: Let $t=|P| \geq 5$. Since every $q \in Q$ sees at least $|P|-1$ vertices of $\operatorname{conv}(P)$, the convex hull $\operatorname{conv}(P)$ has either $t-1$ or $t$ vertices. We consider these two cases separately.

Case 1: $\operatorname{conv}(P)$ has $t-1$ vertices. In this case, every point $q \in Q$ sees all vertices of $\operatorname{conv}(P)$. Pick an arbitrary point $q_{0} \in Q$. Label the points in $P$ such that $\operatorname{conv}(P)=\left(p_{1}, \ldots, p_{t-1}\right)$ where $q_{0} p_{1}$ and $q_{0} p_{t-1}$ are tangent to $\operatorname{conv}(P)$. Refer to Fig. 5 (left). Let $P^{\prime}=\left\{p_{2}, \ldots, p_{t-2}\right\}$. It is clear that $P^{\prime}$ is in convex 
position and every point $q \in Q$ sees all vertices of $\operatorname{conv}\left(P^{\prime}\right)$. It remains to show that $P^{\prime}$ avoids $Q$.

First assume that $t=5$. In this case, $P^{\prime}=\left\{p_{2}, p_{3}\right\}$, and $\operatorname{conv}\left(P^{\prime}\right)$ is the line segment $p_{2} p_{3}$. Let $\ell_{1}$ be a directed line spanned by $p_{2} p_{3}$ such that $q_{0} \in \ell^{+}$ and $p_{1}, p_{2} \in \ell^{-}$. For every point $q^{\prime} \in \ell^{-}$, the segment $p_{2} q$ or $p_{3} q$ intersects the interior of $\operatorname{conv}(P)$. Consequently, every point $q \in Q$ lies in $\ell^{+}$, and so $P^{\prime}$ avoids $Q$.

Assume now that $t \geq 6$. Let $\ell_{1}$ and $\ell_{2}$ be the supporting lines of $p_{2} p_{3}$ and $p_{t-3} p_{t-2}$, respectively. Lines $\ell_{1}$ and $\ell_{2}$ subdivide the plane into 4 wedges. Orient the lines $\ell_{1}$ and $\ell_{2}$ such that $q$ lies in the wedge $\ell_{1}^{+} \cap \ell_{2}^{+}$. Every point $q^{\prime}$ in $\ell_{1}^{+} \cap \ell_{2}^{+}$ sees the vertices of $\operatorname{conv}\left(P^{\prime}\right)$ in the same circular order. If $q^{\prime} \in \ell_{1}^{+} \cap \ell_{2}^{-}$(resp., $q^{\prime} \in \ell_{1}^{-} \cap \ell_{2}^{+}$) then $p_{t-2} q^{\prime}$ (resp., $\left.p_{1} q^{\prime}\right)$ intersects the interior of $\operatorname{conv}(P)$. For every point $q^{\prime} \in \ell_{1}^{-} \cap \ell_{2}^{-}$, segment $p_{2} q^{\prime}$ intersects the interior of $\operatorname{conv}(P)$. Hence, $Q \subset \ell_{1}^{+} \cap \ell_{2}^{+}$. Therefore, every point $q^{\prime} \in Q$ sees the vertices of $\operatorname{conv}\left(P^{\prime}\right)$ in the same circular order, and so $P^{\prime}$ avoids $Q$. This proves part (i).

For part (ii), we set $P^{\prime \prime}=\left\{p_{1}, \ldots, p_{t-1}\right\}$. For every $q \in Q$, consider the counterclockwise order of the points in $P^{\prime \prime}$ between two tangent lines from $q$ to $\operatorname{conv}\left(P^{\prime \prime}\right)$. Since $Q \subseteq \ell_{1}^{+} \cap \ell_{2}^{+}$, this order is $\left(p_{1}, \ldots, p_{t-1}\right),\left(p_{2}, \ldots, p_{t-1}, p_{1}\right)$ or $\left(p_{t-1}, p_{1}, \ldots, p_{t-2}\right)$. Partition $Q$ into three subsets based on the order in which they see the vertices of $\operatorname{conv}\left(P^{\prime \prime}\right)$. Let $Q^{\prime \prime}$ be the largest subset of $Q$, of size at least $\lceil|Q| / 3\rceil$. Now the point set $P^{\prime \prime}$ avoids $Q^{\prime \prime}$, as required.

Case 2: $\operatorname{conv}(P)$ has $t$ vertices. Let $\operatorname{conv}(P)=\left(p_{1}, \ldots, p_{t}\right)$ and let $B \subseteq P$ be the set of points $p_{i} \in P$ such that $p_{i} q$ intersects the interior of $\operatorname{conv}(P)$ for some $q \in Q$. If $B=\emptyset$, we can take $P^{\prime}=P$, and then $P^{\prime}$ avoids $Q$. If $|B|=1$, then the proof of part (i) is analogous to Case 1 with $B=\left\{p_{t}\right\}$.

Assume that $|B| \geq 2$. We show that for any two points in $B$, the hop distance along $\operatorname{conv}(P)$ is at most 2 . It will follow that $|B| \leq 3$. Suppose, to the contrary, that $p_{1}, p_{i} \in B$, where $4 \leq i \leq t-2$. Then there exist points $q_{1}, q_{i} \in Q$ such that both $p_{1} q_{1}$ and $p_{i} q_{i}$ intersect the interior of $\operatorname{conv}(P)$. Let $\ell_{3}$ be the supporting line of $p_{2} p_{3}$ such that $p_{1} \in \ell_{3}^{+}$. Refer to Fig. 5 (right). We have $q_{1}, q_{i} \in \ell_{3}^{-}$, since the line segments between $\left\{q_{1}, q_{i}\right\}$ and $\left\{p_{2}, p_{3}\right\}$ cannot cross the interior of $\operatorname{conv}(P)$ by our assumptions. Let $p_{j}$ be the vertex of the convex chain $\left(p_{i+1}, \ldots, p_{t}\right)$ that lies farthest from the line $\ell_{3}$. If $j=t$, then the segment $p_{j-1} q_{i}$ intersects the interior of $\operatorname{conv}(P)$. If $j<t$, then $p_{j+1} q_{1}$ intersects the interior of $\operatorname{conv}(P)$. That is, the point $q_{1}$ or $q_{i}$ sees at most $t-1$ vertices of $\operatorname{conv}(P)$, contradicting our assumptions. It follows that $|B| \leq 3$ since the hop distance between any two points in $B$ is at most two, and $t \geq 5$. Let $P^{\prime}=P \backslash B$ of size $\left|P^{\prime}\right| \geq|P|-3$. Now $P^{\prime}$ avoids $Q$ by the definition of $B$. This completes the proof of part (i).

For part (ii), notice that every point $q \in Q$ sees at least $|P|-1$ vertices of $\operatorname{conv}(P)$, namely is sees the vertices in $P \backslash\{b\}$ for some $b \in B$. Since $|B| \leq 3$, we can partition the points in $Q$ into at most three subsets based on which vertices of $\operatorname{conv}(P)$ they see. Let $Q^{\prime \prime}$ be a largest subset of $Q$, and $P^{\prime \prime}$ the corresponding set $P \backslash\{b\}, b \in B$, that they all see. Then the $P^{\prime \prime} \subseteq P$ has size $t-1$ and it avoids the set $Q^{\prime \prime}$ of size at least $\lceil|Q| / 3\rceil$. 
Proposition 5 Let $P$ and $Q$ be disjoint point sets in the plane such that $P$ is in convex position, every point $q \in Q$ sees all vertices of $\operatorname{conv}(P)$, and $P$ avoids $Q$. Then, there exist subsets $P^{\prime} \subset P$ and $Q^{\prime} \subset Q$ of size $\left|P^{\prime}\right| \geq\lceil|P| / 2\rceil$ and $\left|Q^{\prime}\right| \geq\lceil\sqrt{|Q|}\rceil$ such that $P^{\prime}$ and $Q^{\prime}$ are mutually avoiding.
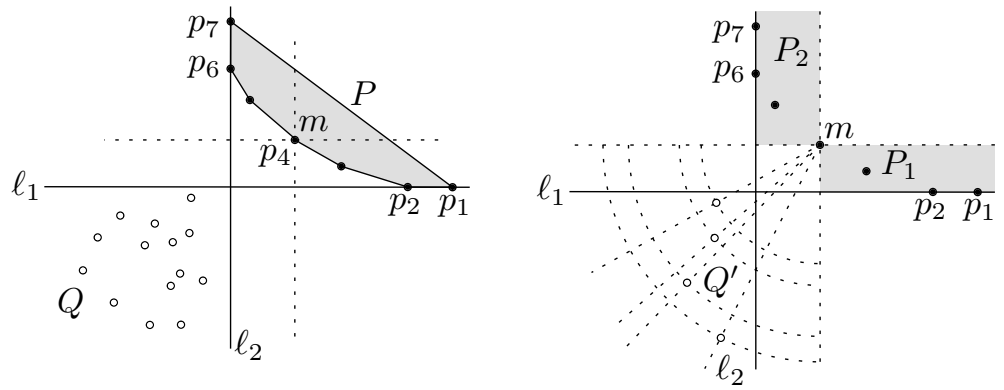

Figure 6: Left: Every point $q \in Q$ sees the same convex $\operatorname{arc}\left(p_{1}, \ldots, p_{7}\right)$ on the boundary of $\operatorname{conv}(P)$. The supporting lines of $p_{1} p_{2}$ and $p_{6} p_{7}$ are $\ell_{1}$ and $\ell_{2}$, respectively. Right: A subsequence $Q^{\prime}=\left(q_{k_{i}}\right)$ whose angles $\theta_{k_{i}}$ about $m$ are monotonically increasing.

Proof: Let $t=|P|$. Since $P$ avoids $Q$, every point $q \in Q$ sees the same convex arc on the boundary of $\operatorname{conv}(P)$, say $\left(p_{1}, \ldots, p_{t}\right)$ in counterclockwise order. Let $\ell_{1}$ and $\ell_{2}$ be the supporting lines of $p_{1} p_{2}$ and $p_{t-1} p_{t}$, respectively. Refer to Fig. 6 . The points in $Q$ must lie in the wedge between $\ell_{1}$ and $\ell_{2}$ not containing points of $P$. We may assume (after performing an appropriate affine transformation) that $\ell_{1}$ and $\ell_{2}$ are orthogonal and parallel to the coordinate axes, $P$ lies in the 1st quadrant and $Q$ lies in the 3rd quadrant.

The midpoint $m$ of the (possibly degenerate) line segment $p_{\lceil t / 2\rceil} p_{\lceil(t+1) / 2\rceil}$ decomposes the convex arc $\left(p_{1}, \ldots, p_{t}\right)$ into two arcs: $P_{1}=\left(p_{1}, \ldots, p_{\lceil t / 2\rceil}\right)$ and $P_{2}=\left(p_{\lceil(t+1) / 2\rceil}, \ldots, p_{t}\right)$. Consider the points in $Q$ written in polar coordinates $(r, \theta)$ where $m$ is the origin and $\theta$ is measured counterclockwise. Order the points $q_{i}=\left(r_{i}, \theta_{i}\right) \in Q$ in decreasing distance $r_{i}$ from $m$. By the Erdős-Szekeres theorem [15], there is a subsequence $Q^{\prime}=\left(q_{k_{i}}\right)$ of length at least $\lceil\sqrt{|Q|}\rceil$ whose angles $\theta_{k_{i}}$ are in either increasing or decreasing order. If they are increasing, we show that $Q^{\prime}$ avoids $P_{1}$. (Analogously, one can show that $Q^{\prime}$ avoids $P_{2}$ if the angles are decreasing.) Consider two points $q_{k_{i}}, q_{k_{j}} \in Q^{\prime}$ with $i<j$. Then $q_{k_{j}}$ lies below the supporting line of $m q_{k_{i}}$ farther from $m$ than $q_{k_{i}}$. Therefore, the supporting line of $q_{k_{i}} q_{k_{j}}$ intersects the vertical line through $m$ either above $m$ or below the $x$-axis $\ell_{1}$. Since all points in $P_{1}$ lie to the right of $m$, in a horizontal strip between $m$ and $\ell_{1}$, the supporting line of $q_{k_{i}} q_{k_{j}}$ avoids $\operatorname{conv}\left(P_{1}\right)$, as claimed.

Proposition 6 Let $P$ and $Q$ be two convexly avoiding sets of size $|P| \geq 3$ and $|Q| \geq 3$. Then there exist subsets $P^{\prime} \subseteq P$ and $Q^{\prime} \subset Q$ such that $\left|P^{\prime}\right|+\left|Q^{\prime}\right| \geq$ $|P|+|Q|-1$, and $P^{\prime}$ and $Q^{\prime}$ are mutually avoiding. 


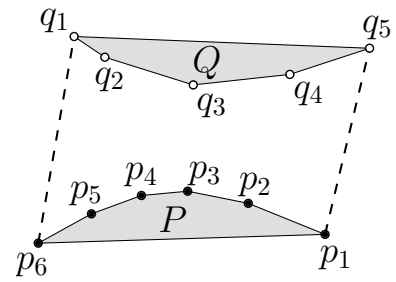

(a)

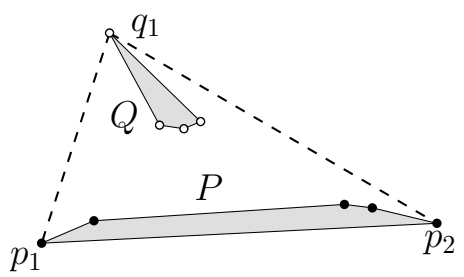

(b)

Figure 7: (a) $\operatorname{conv}(P \cup Q)$ is a quadrilateral. (b) $\operatorname{conv}(P \cup Q)$ is a triangle.

Proof: Since $P$ and $Q$ are convexly avoiding, the convex hulls $\operatorname{conv}(P)$ and $\operatorname{conv}(Q)$ are disjoint, and so $\operatorname{conv}(P)$ and $\operatorname{conv}(Q)$ have exactly two common external tangents. No three consecutive vertices of $\operatorname{conv}(P \cup Q)$ are in $P$ or $Q$, otherwise the middle vertex would be incident to an edge $p_{i} q_{i}$ that intersects the interior of $\operatorname{conv}(P)$ or $\operatorname{conv}(Q)$. Therefore, $\operatorname{conv}(P \cup Q)$ is either a triangle or a quadrilateral.

Assume first that $\operatorname{conv}(P \cup Q)$ is a quadrilateral (Fig. 7(a) . In this case, we show that $P$ and $Q$ are mutually avoiding. Let $\operatorname{conv}(P)=\operatorname{conv}\left(p_{1}, \ldots, p_{t}\right)$ in counterclockwise order such that $p_{t} p_{1}$ lies on the boundary of $\operatorname{conv}(P \cup Q)$. Suppose, to the contrary, that there are two points $p_{i}, p_{j} \in P, 1 \leq i<j \leq t$, such that the supporting line of $p_{i} p_{j}$ intersects conv $(Q)$. Clearly, $p_{i} p_{j} \neq p_{1} p_{t}$, and we may assume (by applying a reflection if necessary) that $j<t$. The line $p_{i} p_{j}$ intersects two edges of $\operatorname{conv}(Q)$. One of them is an edge of $\left.\operatorname{conv}(P \cup Q)\right)$,

and we denote the other one by $q_{k} q_{k+1}$. Then the segments $p_{i} p_{t}, p_{i} q_{k}, p_{i} p_{j}$, and $p_{i} q_{k+1}$ appear in this counterclockwise order around $p_{i}$. Therefore, segment $p_{i} q_{k}$ intersects the interior of $\operatorname{conv}(P)$, contradicting our assumption that $P$ and $Q$ are convexly avoiding.

Assume now that $\operatorname{conv}(P \cup Q)$ is a triangle. Without loss of generality, we may assume $\operatorname{conv}(P \cup Q)=\left(p_{1}, p_{2}, q_{1}\right)$. In this case, $\operatorname{conv}\left(P \cup\left(Q \backslash\left\{q_{1}\right\}\right)\right)$ is a quadrilateral (Fig. $7(\mathrm{~b}))$. The previous argument readily implies that $P$ and $Q \backslash\left\{q_{1}\right\}$ are mutually avoiding.

\subsection{From One Triangle to Another}

In this subsection, we show that if we already know the position of 8 vertices of a complete graph, then the crossing angle pattern uniquely determines the position of all but at most 4 remaining vertices. It is enough to argue about the position of one new vertex at a time.

Proposition 7 Let $G=K_{n}$ be a complete graph on the vertex set $V=P \cup Q$, with $|P| \geq 3$ and $|Q| \geq 3$, and let $\alpha$ be a crossing angle pattern. Consider a straight-line drawing compatible with $\alpha$ such that $P$ is in convex position, every point $q \in Q$ sees all vertices of $\operatorname{conv}(P)$, and $P$ and $Q$ are mutually avoiding. Then the position of the vertices in $P$ uniquely determines the position of at least one vertex in $Q$. 
Proof: Since $P$ and $Q$ are mutually avoiding, every point $q \in Q$ sees the vertices of $\operatorname{conv}(P)$ in the same (counterclockwise) order. Let $p_{1}, p_{2}, p_{3} \in P$ be three arbitrary points in $P$, labeled such that every $q \in Q$ sees $p_{1}, p_{2}$, and $p_{3}$ in this (counterclockwise) order. Similarly, let $q_{1}, q_{2}, q_{3} \in Q$ be three arbitrary points in $Q$ such that every $p \in P$ sees them in this (counterclockwise) order. Refer to Fig. 8(a). The edge $p_{1} q_{1}$ crosses edges $q_{2} p_{2}$ and $q_{2} p_{3}$, and these three edges bound a triangular region in the plane. Since the crossing angles are known, $\angle p_{2} q_{2} p_{3}$ is uniquely determined. Similarly, using edge $p_{3} q_{3}$, angle $\angle p_{1} q_{2} p_{2}$ is uniquely determined.

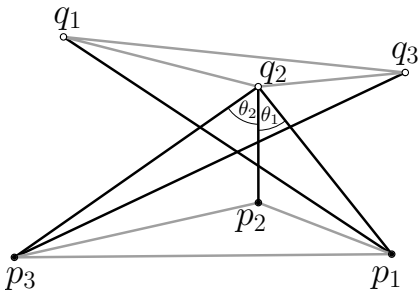

(a)

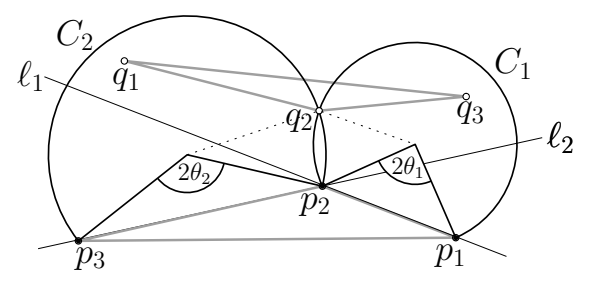

(b)

Figure 8: (a) Determining $\angle p_{2} q_{2} p_{3}$. (b) Inscribed angles.

Let $\ell_{1}$ and $\ell_{2}$ be the supporting lines of $p_{1} p_{2}$ and $p_{2} p_{3}$, respectively. The lines $\ell_{1}$ and $\ell_{2}$ subdivide the plane into 4 wedges. Direct the lines $\ell_{1}$ and $\ell_{2}$ such that $Q$ lies in $\ell_{1}^{+} \cap \ell_{2}^{+}$(Fig. 8(b) . We use a fact from elementary geometry: given two points $a$ and $b$ and an angle $\theta \in(0, \pi)$, the locus of points $c$ with $\angle a b c=\theta$ is the union of two circular arcs that lie on opposite sides of the supporting line of $a b$. We invoke this result with $c=q_{2}$ twice: once for $a b=p_{1} p_{2}$ and once for $a b=p_{2} p_{3}$. Since $q_{2} \in \ell_{1}^{+} \cap \ell_{2}^{+}$, it is enough to use one of the two circular arcs in each invocations. Thus, $q_{2}$ lies on the intersection of two circles: $C_{1}$ defined by $p_{1}, p_{2}, \angle p_{1} q_{2} p_{2}$, and $C_{2}$ defined by $p_{2}, p_{3}, \angle p_{2} q_{2} p_{3}$. Two distinct circles intersect in at most two points. Since $p_{2} \in C_{1} \cap C_{2}$, the position of $q_{2}$ is uniquely determined unless $C_{1}=C_{2}$. If $C_{1}=C_{2}$, then $p_{1}, p_{2}, p_{3}$, and $q_{2}$ all lie on a common circle. This case, however, cannot occur, since it would contradict our assumption that $P$ and $Q$ are convexly avoiding.

Lemma 1 Let $G$ be the complete graph on the vertex set $V=P \cup Q$ such that $|P| \geq 8$ and $|Q| \geq 5$. Let $\alpha$ be a crossing angle pattern. In a straight-line drawing compatible with $\alpha$, the position of the vertices in $P$ uniquely determines the position of at least one vertex in $Q$.

Proof: The vertex set of $G$ is $P \cup Q$, with $|P| \geq 8$ and $|Q| \geq 5$, where the position of the vertices in $P$ are known. If there is a vertex $q \in Q$ such that edges $p_{1} q, p_{2} q$ cross some edges induced by $P$ for some $p_{1}, p_{2} \in P$, then the position of $q$ is determined by Proposition 1. Otherwise, every vertex $q \in Q$ is crossing free or almost crossing free in the subgraph induced by $P \cup\{q\}$. By Proposition 2 or 3. every $q \in Q$ convexly avoids a subset of at least $|P|-1$ points in $P$. By 
Propositions $4(\mathrm{i})$, there is a subset $P^{\prime} \subset P$ of size $8-3=5$ such that every $q \in Q$ sees all vertices of $\operatorname{conv}\left(P^{\prime}\right)$ and $P^{\prime}$ avoids $Q$. By Propositions 5 there are mutually avoiding subsets $P^{\prime \prime} \subseteq P$ and $Q^{\prime \prime} \subseteq Q$, with $\left|P^{\prime \prime}\right| \geq\lceil 5 / 2\rceil=3$ and $\left|Q^{\prime \prime}\right| \geq\lceil\sqrt{5}\rceil=3$, such that every $q \in Q^{\prime \prime}$ convexly avoids $P^{\prime \prime}$. By Proposition 7 . the position of at least one point $q \in Q^{\prime \prime}$ is uniquely determined by the position of $P^{\prime \prime} \subset P$ and the crossing angle pattern $\alpha$.

\subsection{Complete Graphs Are Globally Crossing Angle Rigid}

In Proposition 1 and in Subsection 3.2, we determined the position of a new vertex assuming that we already know the position of some other vertices. We can now drop this assumption and show (Theorem 2 that in complete graph $K_{n}, n \geq 24$, the directed crossing angles always determine the position of at least $n-4$ vertices up to similarity.

Proposition 8 Let $G=K_{n}$ be the complete graph on $n$ vertices, and let $\alpha$ be a crossing angle pattern. If the $n$ vertices form a convex polygon $P$ in a straightline drawing compatible with $\alpha$, then the slope of any diagonal of $P$ determines the slopes of all diagonals of $P$.

Proof: Assume that we are given the slope of a diagonal $e$ of $P$, and all directed crossing angles between diagonals. Let $e^{\prime}$ be another diagonal of $P$. If $e$ crosses $e^{\prime}$, then their crossing angle determines the slope of $e^{\prime}$. If $e$ and $e^{\prime}$ do not cross (Fig. 9(a) $)$, then there is a third diagonal $f$ that crosses both $e$ and $e^{\prime}$, and the slope of $e^{\prime}$ is determined by the angles $\angle(e, f)$ and $\angle\left(f, e^{\prime}\right)$.

Szekeres and Peters [28] proved, by an exhaustive computer search, that every set of 17 points in the plane, no three of which are collinear, contains 6 points in convex position. Note that it is easy to test whether a set of vertices is in convex position based on the directed crossing angle pattern $\alpha: E^{2} \rightarrow(0, \pi) \cup\{\star\}$. Indeed, $\left(p_{1}, \ldots, p_{k}\right)$ is a convex polygon in a straight-line embedding compatible with $\alpha$ if and only if $\alpha\left(p_{i} p_{j}, p_{i^{\prime}} p_{j^{\prime}}\right) \neq \star$ whenever the indices $\left(i, j, i^{\prime}, j^{\prime}\right)$ cross combinatorially in the cyclic sequence $(1, \ldots, k)$.

Theorem 2 Let $G=K_{n}$ be a complete graph with $n \geq 24$ vertices, and let $\alpha$ be directed crossing angle pattern. Then in every straight-line drawing compatible with $\alpha$, the position of at least $n-4$ vertices of $G$ are uniquely determined up to similarity.

Proof: Denote by $V$ the vertex set of $G$. Since all vertex pairs are adjacent, no three vertices are collinear in a straight-line drawing of $G$. Since $|V| \geq 17+6$, we can successively choose two sets, $P \subset V$ and $Q \subseteq(V \backslash P)$, each consisting of 6 points in convex position using [28. Let $\operatorname{conv}(P)=\left(p_{1}, \ldots, p_{6}\right)$ and $\operatorname{conv}(Q)=$ $\left(q_{1}, \ldots, q_{6}\right)$.

By Proposition 8 , the directed crossing angles determine the slopes of all diagonals of a convex hexagon up to similarity. For instance, if we fix two arbitrary vertices in either $\Delta\left(p_{1}, p_{3}, p_{5}\right)$ or $\Delta\left(p_{2}, p_{4}, p_{6}\right)$, then the third vertex 


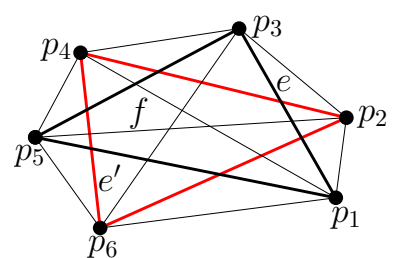

(a)

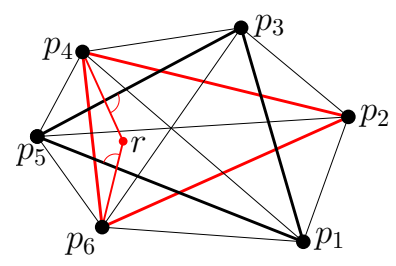

(c)

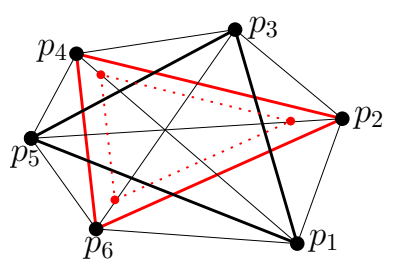

(b)

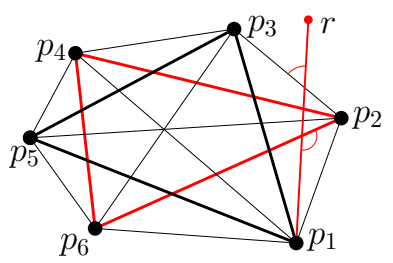

(d)

Figure 9: (a) Diagonals of a convex hexagon. (b) If $\Delta p_{1} p_{3} p_{5}$ is fixed, $\Delta p_{2} p_{4} p_{6}$ has one degree of freedom. (c) A point $r$ lies in the interior of $\operatorname{conv}(P)$. (d) A point $r$ lies in the exterior $\operatorname{conv}(P)$ but does not see all vertices of $\operatorname{conv}(P)$.

of the triangle is uniquely determined. However, if we fix $\Delta\left(p_{1}, p_{3}, p_{5}\right)$, then the vertices of $\Delta\left(p_{2}, p_{4}, p_{6}\right)$ are not necessarily determined (Fig. 9(b)]. We first show that the directed crossing angles determine the position of six points from $P \cup Q$ up to similarity. We distinguish between three cases.

Case 1: There is a vertex $r \in V$ lying in the interior of $\operatorname{conv}(P)$ or $\operatorname{conv}(Q)$. Assume without loss of generality that $r$ lies in the interior of $\operatorname{conv}(P)$ (Fig. 9(c)). Fix the position of $p_{1}$ and $p_{3}$. This immediately determines the position of $p_{5}$. Note that at least two edges in $\left\{p_{1} r, p_{3} r, p_{5} r\right\}$ cross some edges of the triangle $\Delta\left(p_{2}, p_{4}, p_{6}\right)$. Therefore, by Proposition 1 the position of $r$ is uniquely determined. The position of $p_{1}, p_{3}, p_{5}$, and $r$ uniquely determine $p_{2}, p_{4}$, and $p_{6}$, by repeatedly applying Proposition 1 .

Case 2: There is a vertex $r \in V$ such that $r$ lies in the exterior of $\operatorname{conv}(P)$ and an edge from $r$ to $P$ intersects the interior of $\operatorname{conv}(P)$, or $r$ lies in the exterior of $\operatorname{conv}(Q)$ and an edge from $r$ to $Q$ intersects the interior of $\operatorname{conv}(Q)$. Assume without loss of generality that $p_{1} r$ crosses the interior of $\operatorname{conv}(P)$ (Fig. $9(\mathrm{~d})$ ). Since $p_{1}$ enters the interior of $\operatorname{conv}(P)$, it crosses some diagonals of $\operatorname{conv}(P)$. Fix the position of $p_{1}$ and $p_{3}$. This immediately determines the position of $p_{5}$. The location of $p_{1}$ and the directed crossing angles with a diagonal of $\operatorname{conv}(P)$ determine the supporting line of $p_{1} r$. The supporting line of $p_{1} r$ crosses some edge of $\operatorname{conv}(P)$, which is incident to a vertex $p_{j}, j \in\{2,4,6\}$. Now the position of $p_{j}$ is determined by Proposition 1. By repeatedly applying Proposition 1, we determine the position of $p_{2}, p_{4}$, and $p_{6}$.

Case 3: Both $\operatorname{conv}(P)$ and $\operatorname{conv}(Q)$ have empty interiors, every vertex in the exterior of $\operatorname{conv}(P)$ sees all vertices of $\operatorname{conv}(P)$, and every vertex in the exterior 
of $\operatorname{conv}(Q)$ sees all vertices of $\operatorname{conv}(Q)$. In this case, $P$ and $Q$ are convexly avoiding. By Proposition 6, $P$ and $Q$ have mutually avoiding subsets $P^{\prime} \subset P$ and $Q^{\prime} \subset Q$ of total size $\left|P^{\prime}\right|+\left|Q^{\prime}\right| \geq 11$. Without loss of generality, $P^{\prime}=$ $\left\{p_{1}, \ldots, p_{5}\right\}$ and $Q^{\prime}=\left\{q_{1}, \ldots, q_{6}\right\}$ are mutually avoiding. Fix the position of $p_{1}$ and $p_{3}$, which immediately determines the position of $p_{5}$. By Proposition 7 . the position of $\left\{p_{1}, p_{3}, p_{5}\right\}$ determines the position of at least one vertex in each 3 -element subset of $Q^{\prime}$. Hence, $\left\{p_{1}, p_{3}, p_{5}\right\}$ determines the position of at least 3 vertices in $Q^{\prime}$.

In all three cases, the position of at least 6 vertices are determined up to similarity. Use Proposition 1 1 successively to determine as many more vertices as possible. Let $A \subset V$ be the set of vertices whose position is already determined up to similarity, and let $B=V \backslash A$. If we already know the position of 8 vertices in $V$, then we can use Lemma 1 to determine the position of all but at most 4 vertices in $V$. It remains to consider the case where $6 \leq|A| \leq 7$.

If $6 \leq|A| \leq 7$, then $|B| \geq 24-7 \geq 17$. Since Proposition 1 is not applicable for the points in $B$, they all see at least $|A|-1 \geq 5$ vertices of $\operatorname{conv}(A)$ by Propositions 2 and 3. By Proposition 4(ii), there is a subset $A^{\prime} \subseteq A$ of size $\left|A^{\prime}\right| \geq|A|-1 \geq 5$ in convex position and a subset $B^{\prime} \subseteq B$ of size $\left|B^{\prime}\right| \geq$ $\lceil 17 / 3\rceil=6$ such that $A^{\prime}$ avoids $B^{\prime}$ and every point $b \in B^{\prime}$ sees all vertices of $\operatorname{conv}\left(A^{\prime \prime}\right)$. By Propositions 5 there are mutually avoiding subsets $A^{\prime \prime} \subseteq A^{\prime}$ and $B^{\prime \prime} \subseteq B^{\prime}$, with $\left|A^{\prime \prime}\right| \geq\lceil 5 / 2\rceil=3$ and $\left|B^{\prime \prime}\right| \geq\lceil\sqrt{6}\rceil=3$, such that every $b \in B^{\prime \prime}$ sees all vertices of $\operatorname{conv}\left(A^{\prime \prime}\right)$. By Proposition 7, the position of some point $b \in B^{\prime \prime}$ is uniquely determined. Thus $A$ can be incremented until $|A| \geq 8$.

\subsection{Globally Angle-Rigid Graphs of Bounded Degree}

For every $n \geq 24$, we construct a globally crossing angle rigid graph $F=(V, E)$ with $n$ vertices, bounded vertex degree, and $O(\log n)$ diameter. Refer to Fig. 10 We start with an auxiliary graph $F_{0}$ on a vertex set $V_{0}=\left\{v_{1}, \ldots, v_{n_{0}}\right\}$, for some fixed $n_{0} \geq 2$. Let $F_{0}$ be a binary tree of diameter $O\left(\log n_{0}\right)$. The graph $F$ is obtained from $F_{0}$ by replacing each vertex in $V_{0}$ with a clique $K_{12}$, and replacing each edge of $F_{0}$ with a biclique $K_{12,12}$ between the corresponding cliques. (See Fig. 10 for an illustration.) The vertex set of $F$ is $V=\bigcup_{i=1}^{n_{0}} V_{i}$, where $V_{i}$ is a set of 12 vertices corresponding to $v_{i}$. Hence $F$ has $n=12 n_{0}$ vertices, and its maximum degree is $4 \cdot 12-1=47$.

Theorem 3 Let $D_{1}$ be a straight-line drawing of $F=(V, E)$ compatible with a directed crossing angle pattern $\alpha: E^{2} \rightarrow[0, \pi) \cup\{\star\}$. Then there is a subset $V^{\prime}(\alpha) \subset V$ of vertices such that (i) $V^{\prime}(\alpha)$ contains at least 8 vertices from each $V_{i}, 1 \leq i \leq k$, and (ii) for every straight-line drawing $D_{2}$ of $F$ compatible with $\alpha$, a similarity transformation carries all vertices in $V^{\prime}(\alpha)$ to the corresponding vertices in $D_{1}$.

Proof: Applying Theorem 2 for $V_{1} \cup V_{2}$, we find a subset $V_{1}^{\prime} \subset V_{1}$ of size $\left|V_{1}^{\prime}\right|=8$ such that the position of all vertices in $V_{1}^{\prime}$ is determined up to similarity by the 

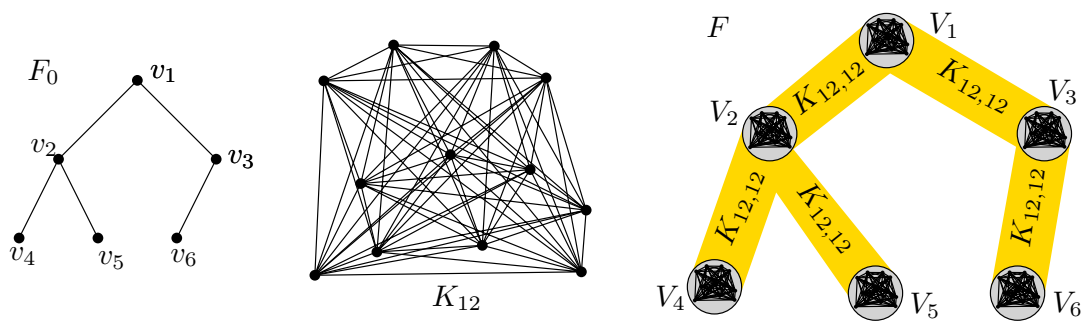

Figure 10: Left: A binary tree $F_{0}$ with $n_{0}=9$ vertices. Middle: A straight-line drawing of $K_{12}$. Right: The graph $F$ constructed from $F_{0}$.

directed crossing angles. We incrementally choose a vertex set $V_{i}^{\prime} \subset V_{i}$ of size $\left|V_{i}^{\prime}\right|=8$ for all $j=2, \ldots, n_{0}$. Assume that we have already chosen a subset $V_{i}^{\prime} \subset V_{i},\left|V_{i}^{\prime}\right|=8$, and $v_{i} v_{j}$ is an edge of the auxiliary graph $F_{0}$. Then $F$ contains a complete graph on the vertex set $V_{i}^{\prime} \cup V_{j}$, where $\left|V_{i}^{\prime}\right|=8$ and $\left|V_{j}\right|=12$. By Lemma 1. we can successively choose 8 elements from $V_{j}$ whose positions are each determined by the position of the vertices in $V_{i}^{\prime}$ and the crossing angles. Denote by $V_{j}^{\prime}$ the set of these elements, with $V_{j}^{\prime} \subset V_{j}$ and $\left|V_{j}^{\prime}\right|=8$. Finally, let $V^{\prime}(\alpha)=\bigcup_{i=1}^{n_{0}} V_{i}^{\prime}$. Note that the crossing angle pattern $\alpha$ determines the position of all vertices in $V^{\prime}(\alpha)$ up to similarity, as claimed.

\section{Conclusions}

Note that the function $\alpha: E^{2} \rightarrow[0, \pi) \cup\{\star\}$ defined above encodes the directed crossing angle pattern. It is natural to consider the undirected crossing angle pattern $\beta:\left(\begin{array}{c}E \\ 2\end{array}\right) \rightarrow(0, \pi / 2) \cup\{\star\}$ for unordered pairs $\{e, f\} \in\left(\begin{array}{c}E \\ 2\end{array}\right)$. Our method is likely to extend to this variant of the problem, and produce results analogous to Theorems 2 and 3), with higher vertex degrees. We have not pursued this direction in this paper.

Our results represent a first step towards a possible combinatorial characterization of globally crossing angle rigid graphs. In our definition of globally crossing angle rigid graphs, a directed crossing angle pattern $\alpha$ determines at least half of the vertices up to similarity. In fact, Theorem 3 holds with factor $\frac{2}{3}$ in place of one half. For every constant $c \in(0,1)$, there is a threshold $\Delta(c)$ such that there exist infinitely many graphs $G=(V, E)$ of maximum degree $\Delta(c)$ where a directed crossing angle pattern $\alpha$ determines at least $c|V|$ vertices up to similarity. It is left for future research to find the minimum value of the threshold $\Delta(c)$. Several related problems remain open. Is it NP-hard to decide whether a given straight-line drawing of a graph $G=(V, E)$ is angle-rigid? Is it NP-hard to find the crossing angle number $\operatorname{can}(G)$ of a given graph $G$ ? We do not even know whether the bound $\operatorname{can}\left(K_{n}\right) \leq\lfloor n / 2\rfloor-1$ in Observation 2 is tight for $n \geq 7$. 
418 Arikushi and Tóth Crossing Angles of Geometric Graphs

\section{References}

[1] B. M. Ábrego, S. Fernández-Merchant, and G. Salazar. The rectilinear crossing number of $K_{n}$ : Closing in (or are we?). In J. Pach, editor, Thirty essays in geometric graph theory, pages 5-18. Springer, 2013. doi:10. 1007/978-1-4614-0110-0_2.

[2] E. N. Argyriou, M. A. Bekos, and A. Symvonis. The straight-line RAC drawing problem is NP-hard. J. Graph Algorithms and Applications, 16(2):569-597, 2012. doi:10.7155/jgaa.00274.

[3] K. Arikushi and C. D. Tóth. Crossing angles of geometric graphs. In G. Lin, editor, Combinatorial Optimization and Applications, volume 7402 of $L N C S$, pages 103-114. Springer, 2012. doi:10.1007/ 978-3-642-31770-5_10.

[4] B. Aronov, P. Erdős, W. Goddard, D. J. Kleitman, M. Klugerman, J. Pach, and L. J. Schulman. Crossing families. Combinatorica, 14(2):127-134, 1994. doi:10.1007/BF01215345.

[5] J. Barát, J. Matoušek, and D. R. Wood. Bounded-degree graphs have arbitrarily large geometric thickness. Electronic J. Combinatorics, 13(1):R3, 2006. URL: http://www. combinatorics.org/ojs/index.php/ eljc/article/view/v13i1r3.

[6] D. Bienstock and N. Dean. Bounds for rectilinear crossing numbers. J. Graph Theory, 17(3):333-348, 1993. doi:10.1002/jgt.3190170308.

[7] E. Di Giacomo, W. Didimo, P. Eades, and G. Liotta. 2-layer right angle crossing drawings. Algorithmica, 68(4):954-997, 2014. doi:10.1007/ s00453-012-9706-7.

[8] E. Di Giacomo, W. Didimo, G. Liotta, and H. Meijer. Area, curve complexity, and crossing resolution ofnon-planar graph drawings. Theory of Computing Systems, 49(3):565-575, 2011. doi:10.1007/s00224-010-9275-6.

[9] W. Didimo, P. Eades, and G. Liotta. A characterization of complete bipartite RAC graphs. Information Processing Letters, 110(16):687-691, 2010. doi:10.1016/j.ipl.2010.05.023.

[10] W. Didimo, P. Eades, and G. Liotta. Drawing graphs with right angle crossings. Theoretical Computer Science, 412(39):5156-5166, 2011. doi: $10.1016 / j . t c s .2011 .05 .025$

[11] M. B. Dillencourt, D. Eppstein, and D. S. Hirschberg. Geometric thickness of complete graphs. J. Graph Algorithms and Applications, 4(3):5-17, 2000. doi:10.7155/jgaa.00023.

[12] V. Dujmovi, M. Suderman, and D. R. Wood. Graph drawings with few slopes. Computational Geometry, 38(3):181-193, 2007. doi:10.1016/j. comgeo.2006.08.002 
[13] V. Dujmović, J. Gudmundsson, P. Morin, and T. Wolle. Notes on large angle crossing graphs. Chicago Journal of Theoretical Computer Science, 2011. doi:10.4086/cjtcs.2011.004.

[14] P. Eades and G. Liotta. Right angle crossing graphs and 1-planarity. Discrete Applied Mathematics, 161(78):961-969, 2013. doi:10.1016/j.dam. 2012.11.019.

[15] P. Erdős and G. Szekeres. A combinatorial problem in geometry. In I. Gessel and G.-C. Rota, editors, Classic Papers in Combinatorics, Modern Birkhäuser Classics, pages 49-56. Birkhäuser, 1987. doi:10.1007/ 978-0-8176-4842-8_3.

[16] E. D. Giacomo, W. Didimo, G. Liotta, and F. Montecchiani. Area requirement of graph drawings with few crossings per edge. Computational Geometry, 46(8):909-916, 2013. doi:10.1016/j.comgeo.2013.03.001.

[17] W. Huang, P. Eades, and S.-H. Hong. Larger crossing angles make graphs easier to read. Journal of Visual Languages 83 Computing, 25(4):452-465, 2014. doi:10.1016/j.jvlc.2014.03.001.

[18] B. Jackson and T. Jordán. Connected rigidity matroids and unique realizations of graphs. Journal of Combinatorial Theory, Series B, 94(1):1-29, 2005. doi:10.1016/j.jctb.2004.11.002.

[19] B. Jackson and T. Jordán. Globally rigid circuits of the directionlength rigidity matroid. Journal of Combinatorial Theory, Series B, 100(1):1-22, 2010. doi:10.1016/j.jctb.2009.03.004.

[20] B. Jackson, T. Jordan, and Z. Szabadka. Globally linked pairs of vertices in equivalent realizations of graphs. Discrete $\mathcal{E}$ Computational Geometry, 35(3):493-512, 2006. doi:10.1007/s00454-005-1225-8.

[21] B. Jackson, T. Jordán, and Z. Szabadka. Globally linked pairs of vertices in rigid frameworks. In R. Connelly, A. Ivić Weiss, and W. Whiteley, editors, Rigidity and Symmetry, volume 70 of Fields Institute Communications, pages 177-203. Springer, 2014. doi:10.1007/978-1-4939-0781-6_10.

[22] B. Keszegh, J. Pach, and D. Pálvölgyi. Drawing planar graphs of bounded degree with few slopes. SIAM J. Discrete Math., 27(2):1171-1183, 2013. doi:10.1137/100815001.

[23] P. Mukkamala and D. Plvlgyi. Drawing cubic graphs with the four basic slopes. In M. van Kreveld and B. Speckmann, editors, Graph Drawing, volume 7034 of $L N C S$, pages 254-265. Springer, 2012. doi:10.1007/ 978-3-642-25878-7_25.

[24] J. Pach and D. Pálvölgyi. Bounded-degree graphs can have arbitrarily large slope numbers. Electronic J. Combinatorics, 13(1):N1, 2006. URL: http:// WWw.combinatorics.org/ojs/index.php/eljc/article/view/v13i1n1. 
420 Arikushi and Tóth Crossing Angles of Geometric Graphs

[25] A. Riskin. The crossing number of a cubic plane polyhedral map plus an edge. Studia Scientiarum Mathematicarum Hungarica, 31:405-413, 1996.

[26] F. Saliola and W. Whiteley. Constraining plane configurations in CAD: circles, lines, and angles in the plane. SIAM J. Discrete Math., 18(2):246271, 2004. doi:10.1137/S0895480100374138

[27] J. B. Saxe. Embeddability of weighted graphs in $k$-space is strongly NPhard. In Proc. 17th Allerton Conf. in Communications, Control, and Computing, pages 480-489, 1979.

[28] G. Szekeres and L. Peters. Computer solution to the 17-point ErdösSzekeres problem. ANZIAM J., 48(2):151-164, 2006. URL: http://www. austms.org.au/Publ/Jamsb/V48P2/pdf/2409.pdf.

[29] G. A. Wade and J.-H. Chu. Drawability of complete graphs using a minimal slope set. The Computer Journal, 37(2):139-142, 1994. doi:10.1093/ comjnl/37.2.139.

[30] W. Whiteley. Rigidity and scene analysis. In J. E. Goodman and J. O'Rourke, editors, Handbook of Discrete and Computational Geometry, chapter 60, pages 1327-1354. CRC Press, Boca Raton, FL, 2004. URL: http://www.crcpress.com/product/isbn/9781584883012. 